\title{
ON PATH INTEGRATION ON NONCOMMUTATIVE GEOMETRIES
}

\author{
ACHIM KEMPF \\ Department of Applied Mathematics 83 Theoretical Physics \\ and Corpus Christi College in the University of Cambridge \\ Silver Street, Cambridge CB3 9EW, U.K. \\ E-mail: a.kempf@amtp.cam.ac.uk
}

\begin{abstract}
We discuss a recent approach to quantum field theoretical path integration on noncommutative geometries which imply UV/IR regularising finite minimal uncertainties in positions and/or momenta. One class of such noncommutative geometries arise as 'momentum spaces' over curved spaces, for which we can now give the full set of commutation relations in coordinate free form, based on the Synge world function.
\end{abstract}

1. Introduction. A crucial example of noncommutative geometry [1] is the quantum mechanical phase space with its noncommuting 'coordinate functions' $\mathbf{x}_{i}$ and $\mathbf{p}_{j}$. We investigate the possibility that also the position and momentum spaces acquire noncommutative geometric features, i.e. we consider associative Heisenberg algebras $\mathcal{A}$ generated by elements $\mathbf{x}_{i}, \mathbf{p}_{j}$, now allowing

$$
\left[\mathbf{x}_{i}, \mathbf{x}_{j}\right] \neq 0, \quad\left[\mathbf{p}_{i}, \mathbf{p}_{j}\right] \neq 0
$$

and also:

$$
\left[\mathbf{x}_{i}, \mathbf{p}_{j}\right]=i \hbar\left(\delta_{i j}+\alpha_{i j k l} \mathbf{x}_{k} \mathbf{x}_{l}+\beta_{i j k l} \mathbf{p}_{k} \mathbf{p}_{l}+\ldots\right)
$$

We restrict ourselves to relations that allow the involution $\mathbf{x}_{i}^{*}=\mathbf{x}_{i}, \mathbf{p}_{i}^{*}=\mathbf{p}_{i}$, i.e. for which '*) extends to an anti algebra homomorphism. To motivate the particular form of relation Eq.2, let this relation be represented on a dense domain $D \subset H$ in a Hilbert space $H$, i.e. both the $\mathbf{x}_{i}$ and the $\mathbf{p}_{j}$ are to be represented as symmetric operators on $D$. Assuming, e.g. in the simplest case of one dimension, $\alpha, \beta>0$ and $\alpha \beta<1 / \hbar^{2}$, together with the usual definition of uncertainties

$$
(\Delta x)_{|\psi\rangle}^{2}:=\left\langle\psi\left|(\mathbf{x}-\langle\psi|\mathbf{x}| \psi\rangle)^{2}\right| \psi\right\rangle
$$

1991 Mathematics Subject Classification: Primary 81S05; Secondary 83C47.

The paper is in final form and no version of it will be published elsewhere. 
yields

$$
\Delta x \Delta p \geq \frac{\hbar}{2}\left(1+\alpha(\Delta x)^{2}+\alpha\langle\mathbf{x}\rangle^{2}+\beta(\Delta p)^{2}+\beta\langle\mathbf{p}\rangle^{2}\right)
$$

As is not difficult to check Eq.4 implies that there are finite minimal uncertainties $\Delta x_{0}=$ $\left(1 / \beta \hbar^{2}-\alpha\right)^{-1 / 2}$ and $\Delta p_{0}=\left(1 / \alpha \hbar^{2}-\beta\right)^{-1 / 2}$, so that there appears a 'minimal uncertainty gap' (all $|\psi\rangle$ normalised):

$$
\forall|\psi\rangle \in D: \quad \Delta x_{|\psi\rangle} \geq \Delta x_{0} \quad \text { and } \quad \Delta p_{|\psi\rangle} \geq \Delta p_{0}
$$

Physically, since $\alpha$ and $\beta$ can be assumed small, we have ordinary quantum mechanical behaviour on medium scales. The presence of a finite $\Delta x_{0}$, physically relevant in the ultraviolet, e.g. at the Planck scale, can be motivated from studies in string theory and quantum gravity, see e.g. [2]-[5]. The presence of a finite $\Delta p_{0}$, relevant in the infrared, i.e. on large scales, may be motivated from the absence of plane waves (i.e. of sharp localisations in momentum space) on generic curved spaces, see [10].

It should be interesting to apply A.Connes programme to such generalised phase spaces, i.e. in particular to develop the corresponding differential and integral calculus, though we will here not follow this approach. Instead we will focus on the field theoretic and gravity-related aspects of the ansatz.

2. Representation theory. Due to the minimal uncertainty gap Eq.5, algebras $\mathcal{A}$ which imply minimal uncertainties cannot find spectral representations of neither the $\mathbf{x}$ nor $\mathbf{p}$ (recall that e.g. $\mathbf{x}|\psi\rangle=x|\psi\rangle$ implies $\Delta x_{|\psi\rangle}=0$ ). In fact we give up (essential) self-adjointness of the $\mathbf{x}$ and $\mathbf{p}$ on physical domains $D$ to retain only their symmetry. While giving up self-adjointness is necessary for the description of the new short distance behaviour, the symmetry is sufficient for the definition of uncertainties to be well defined, and to ensure the realness of expectation values, e.g. $\forall|\psi\rangle \in D:\langle\psi|\mathbf{x}| \psi\rangle \in \mathbb{R}$. The situation has been studied in explicit representations. The deficiency indices have, e.g. in one dimension, been found as $(1,1)$ and the self-adjoint extensions and eigenvectors of the $\mathbf{x}$ and $\mathbf{p}$ have been calculated. The spectra of the $\mathbf{x}$ and $\mathbf{p}$ are discrete, with the eigenvectors in $H$, though of course not in $D$. Unlike the case of the ordinary commutation relations there are now no sequences of physical states which would approximate point localisation i.e. $\nexists\left\{\left|\psi_{n}\right\rangle \in D\right\}$ : $\lim _{n \rightarrow \infty}(\Delta x)_{\left|\psi_{n}\right\rangle}=0$ and analogously for the momentum.

We give two examples of Hilbert space representations for the one-dimensional case. With $L, K$ length and momentum scales obeying $L K=\left(q^{2}+1\right) / 4$ and $q>1$ the relation

$$
[\mathbf{x}, \mathbf{p}]=i \hbar\left(1+\left(q^{2}-1\right)\left(\mathbf{x}^{2} / 4 L^{2}+\mathbf{p}^{2} / 4 K^{2}\right)\right)
$$

can be represented e.g. on polynomials in $\bar{\eta}$ through

$$
\mathbf{x} . \psi(\bar{\eta})=L\left(\bar{\eta}+\partial_{\bar{\eta}}\right) \psi(\bar{\eta}) \quad \mathbf{p} \cdot \psi(\bar{\eta})=i K\left(\bar{\eta}-\partial_{\bar{\eta}}\right) \psi(\bar{\eta})
$$

where the differentiations are to be evaluated algebraically by commuting $\partial_{\bar{\eta}}$ to the right using the 'Leibnitz rule'

$$
\partial_{\bar{\eta}} \bar{\eta}-q^{2} \bar{\eta} \partial_{\bar{\eta}}=1
$$

and where the scalar product now reads $\left\langle\psi_{1} \mid \psi_{2}\right\rangle=\left.\psi_{1}^{*}\left(\partial_{\bar{\eta}}\right) \psi_{2}(\bar{\eta})\right|_{\bar{\eta}=0}$. In the special case 
$\alpha=0$ the algebra $\mathcal{A}$ still finds spectral representations of $\mathbf{p}$. A convenient possibility is

$$
\text { p. } \psi(p)=p \psi(p), \quad \mathbf{x} \cdot \psi(p)=i \hbar\left(1+\beta \mathbf{p}^{2}\right) \partial_{p} \psi(p)
$$

with the scalar product $\left\langle\psi_{1} \mid \psi_{2}\right\rangle=\int_{-\infty}^{\infty} d p\left(1+\beta p^{2}\right)^{-1} \psi_{1}^{*}(p) \psi_{2}(p)$. Using the Bargmann Fock representation Eqs.7,8 physical features such as the scalar product of maximal localisation states have been calculated [13] and these results nicely reduce to the results previously obtained working in the momentum representation Eq.9 in the special case $\alpha=0$, see [12]. This is however nontrivial, and for generic algebras $\mathcal{A}$ not all Hilbert space representations will be unitarily equivalent. We conjecture that the unitary equivalence does hold in the sense in which it holds for the ordinary commutation relations, for those cases considered so far, i.e. with positive (matrices) $\alpha, \beta$. For $\alpha$ or $\beta$ negative nonequivalent representations can be found [14].

To the best of my knowledge relations equivalent to the $\bar{\eta}, \partial_{\bar{\eta}}$-relations Eq. 8 were first studied in [15], and the $n$ - dimensional $S U_{q}(n)$ - covariant generalisation was first studied in [16]. The relation Eq.6, its physical implications and that one of its Hilbert space representations is on the algebra of these $\bar{\eta}, \partial_{\bar{\eta}}$ was however not known, see also $[6,7,17]$. That the generalised Heisenberg algebra of positions and momenta with commutation relations Eq.6 (and generalisations) imply finite minimal uncertainties $\Delta x_{0}, \Delta p_{0}$, and the crucial interplay between the functional analysis of the $\mathbf{x}$ and $\mathbf{p}$ was first shown in $[8,9]$. The special case $\alpha=0$ was first studied in [12] and the there obtained results on maximal localisation states have been extended to the general situation in [13]. Our approach to quantum field theory on noncommutative geometries, that we will now describe, was first studied in $[10,11]$.

3. Path integration. Let us illustrate the ansatz with the simple example of charged euclidean $\phi^{4}$-theory. Its partition function

$$
Z[J]:=N \int D \phi e^{\int d^{4} x \phi^{*}\left(\partial_{i} \partial_{i}-\mu^{2}\right) \phi-\frac{\lambda}{4 !}(\phi \phi)^{*} \phi \phi+\phi^{*} J+J^{*} \phi}
$$

we rewrite as

$$
Z[J]=N \int_{D} D \phi e^{-\operatorname{tr}\left(\frac{1^{2}}{\hbar^{2}}\left(\mathbf{p}^{2}+\mathrm{m}^{2} \mathrm{c}^{2}\right) \cdot|\phi\rangle\left\langle\phi\left|+\frac{\lambda 1^{4}}{4 !}\right| \phi * \phi\right\rangle\langle\phi * \phi|+| \phi\rangle\langle\mathrm{J}|+| J\rangle\langle\phi|\right)}
$$

where, to make the units transparent, we introduced an arbitrary positive length to render the fields unitless ( $l$ could trivially be reabsorbed in the fields).

We recover Eq.10 from Eq.11 by assuming the ordinary relations $\left[\mathbf{x}_{i}, \mathbf{p}_{j}\right]=i \hbar \delta_{i j}$ in $\mathcal{A}$ and by choosing the spectral representation of the $\mathbf{x}_{i}$ where with $\phi(x):=\langle x \mid \phi\rangle$

$$
\mathbf{x}_{i} \cdot \phi(x)=x_{i} \phi(x), \quad \mathbf{p}_{i} \cdot \phi(x)=-i \hbar \partial_{x_{i}} \phi(x)
$$

and where

$$
\operatorname{tr}(\mathrm{q})=\int \mathrm{d}^{4} \mathrm{x}\langle\mathrm{x}|\mathrm{q}| \mathrm{x}\rangle, \quad\left(\phi_{1} * \phi_{2}\right)(\mathrm{x})=\phi_{1}(\mathrm{x}) \phi_{2}(\mathrm{x})
$$

The pointwise multiplication $*$, used to express point interaction, is (and can also on noncommutative geometries be kept) commutative for bosons. Since fields are in a representation of $\mathcal{A}$, similar to quantum mechanical states, we formally extended Dirac's bra-ket notation for states to fields. In Eq.11 this yields a convenient notation for the 
functional analytic structure of the action functional, but of course, the quantum mechanical interpretation does not simply extend, see e.g. [18]. The space $D$ of fields that is formally to be summed over can be taken to be the dense domain $S_{\infty}$ in the Hilbert space $H$ of square integrable fields.

Generally, the unitary transformations that map from one Hilbert basis to another have trivial determinant, so that no anomalies are introduced into the field theory and changes of basis can be performed arbitrarily, in the action functional, in the Feynman rules or in the end results of the calculation of $n$ - point functions. Choosing any other, e.g. discrete, Hilbert basis $\{|n\rangle\}$ in $D$ we have $\phi_{n}=\langle n \mid \phi\rangle$ so that

$$
\left(\mathbf{p}^{2}+m^{2}\right)_{n m}=\left\langle n\left|\mathbf{p}^{2}+m^{2}\right| m\right\rangle
$$

and

$$
*=\sum_{n_{i}} L_{n_{1}, n_{2}, n_{3}}\left|n_{1}\right\rangle \otimes\left\langle n_{2}\right| \otimes\left\langle n_{3}\right|
$$

Thus:

$$
Z[J]=N \int_{F} D \phi e^{-\frac{l^{2}}{\hbar^{2}} \phi_{n_{1}}^{*}\left(\mathbf{p}^{2}+m^{2}\right)_{n_{1} n_{2}} \phi_{n_{2}}-\frac{\lambda l^{4}}{4 !} L_{n_{1} n_{2} n_{3}}^{*} L_{n_{1} n_{4} n_{5}} \phi_{n_{2}}^{*} \phi_{n_{3}}^{*} \phi_{n_{4}} \phi_{n_{5}}+\phi_{n}^{*} J_{n}+J_{n}^{*} \phi_{n}}
$$

Pulling the interaction term in front of the path integral, completing the squares, and carrying out the gaussian integrals yields

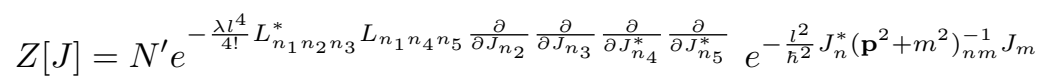

Generally, the inverse of $\left(\mathbf{p}^{2}+m^{2}\right)$ on $D$ may not be unique in which case we choose a self-adjoint extension in which it can be diagonalised and inverted, i.e. physically one chooses boundary conditions. We obtain the Feynman rules:

$$
\Delta_{n_{1} n_{2}}=\left(\frac{\hbar^{2}}{l^{2}\left(\mathbf{p}^{2}+m^{2}\right)}\right)_{n_{1} n_{2}} \quad \text { and } \quad \Gamma_{n_{1} n_{2} n_{3} n_{4}}=-\frac{\lambda l^{4}}{4 !} L_{m n_{1} n_{2}}^{*} L_{m n_{3} n_{4}}
$$

Let us recall that the usual formulation of partition functions, such as e.g. Eq.10, implies that $\mathbf{p}^{2}$ can be represented as the d'Alembertian on a spectral representation of the $\mathbf{x}_{i}$, so that $\mathbf{p}_{i}$ is represented as $-i \hbar \partial_{i}$, i.e. it is implied that $\left[\mathbf{x}_{i}, \mathbf{p}_{j}\right]=i \hbar \delta_{i j}$. It is crucial that in our formulation of partition functions in abstract form, such as in Eq.11, the commutation relations of the underlying algebra $\mathcal{A}$ are not implicitly fixed and can be generalised, e.g. to the form of Eqs.1,2.

Representing $\mathcal{A}$ on some $D$ in a Hilbert space $H$ with an e.g. discrete Hilbert basis $\{|n\rangle\}$ (the Hilbert space is separable), one straightforwardly obtains the Feynman rules through Eqs.16-18. The formalism thus allows for example to explicitly check noncommutative geometries on UV and IR regularisation. So far, regularisation has been shown only for certain examples of geometries $\mathcal{A}$ that imply minimal uncertainties $\Delta x_{0}, \Delta p_{0}$, see $[10]$.

Without going into details, let us only remark that in this context the structure of the pointwise multiplication $*$ that describes local interaction is crucial. Due to the absence of a position representation, it is nonunique in the case of $\Delta x_{0}>0$, though there are still 'quasi-position representations' $[12,13]$, built on maximal localisation states, which 
can be used to establish the locality and causality properties of pointwise multiplications. Generally in our approach an interaction is observationally local if any formal nonlocality of $*$ is not larger than the scale of the nonlocality $\Delta x_{0}$ inherent in the underlying space. Thus, a UV-regular, formally slightly nonlocal $*$, can describe an observationally strictly local interaction. Further studies on locality and regularisation are in progress [14]. An alternative approach, based on the canonical formulation of field theory is [24].

4. Curvature and noncommutativity. Assuming for $\mathcal{A}$ commutation relations of the type of Eqs.1,2 means in particular that the momenta are no longer the generators of what would be infinitesimal translations on flat space, because then $\mathbf{x}_{i} \rightarrow \mathbf{x}_{i}^{\prime}:=$ $\mathbf{x}_{i}-\alpha_{j}\left[\mathbf{x}_{i}, \mathbf{p}_{j}\right] / i \hbar \neq \mathbf{x}_{i}-\alpha_{i}$. We intend to show that certain commutation relations of the type of Eqs.1,2 can arise for momenta on curved space.

Let $\mathcal{M}$ be a (pseudo-) Riemannian manifold where we for simplicity assume vanishing torsion. Due to the path dependence of parallel transport any definition of 4-vectors of positions $\mathbf{x}$ and momenta $\mathbf{p}$ must relate to measurement prescriptions that specify some point $Q$ on the manifold for $\mathbf{x}$ and $\mathbf{p}$ to live in the (co-) tangent spaces to $Q$. Choosing an event $Q \in \mathcal{M}$ there exist normal (i.e. geodesically convex) neighbourhoods $U(Q)$ in which the Synge world function $\sigma$, see e.g. $[22,23]$ is well defined through

$$
\sigma(Q, x):=\frac{1}{2} s^{2}
$$

with $s$ being the geodesic distance between events $Q$ and $x$. Covariant differentiation with respect to $Q$

$$
\sigma^{\mu}(Q, x):=-g^{\mu \nu}(Q) \nabla_{\nu} \sigma(Q, x)
$$

yields the vector field of geodesic coordinates of the event $x$, i.e. $\sigma^{\mu}(Q, x) \in T_{Q}(\mathcal{M})$ is the initial tangent vector of the geodesic that reaches $x$ as its parameter reaches 1 .

We now define for each $Q$ a local Heisenberg algebra $\mathcal{A}_{Q}$, generated by elements $\mathbf{x}_{Q}^{\mu}, \mathbf{p}_{Q \nu}$ which, as vectors, lie in the (co-) tangent spaces to $Q$. We start by defining $\left[\mathbf{x}_{Q}^{\mu}, \mathbf{x}_{Q}^{\nu}\right]=0$ and by mapping the spectrum of the $\mathbf{x}_{Q}^{\mu}$ onto the manifold through the exponential map. Hence the $\mathbf{x}_{Q}^{\mu}$ correspond to the measurement of the coordinates $\sigma^{\mu}$ of the event $x$ in the geodesic coordinate system, or frame, with origin $Q$. The momentum operators now be defined to generate the change of the geodesic coordinates as we move the origin of the geodesic frame from the event $Q$, by $\alpha \in T_{Q}(\mathcal{M})$, to the infinitesimally distant $Q^{\prime}$

$$
\mathbf{x}_{Q}^{\mu} \rightarrow \mathbf{x}_{Q^{\prime}}^{\mu}=\mathbf{x}_{Q}^{\mu}-\alpha^{\nu} \sigma_{\nu}^{\mu}\left(Q, \mathbf{x}_{Q}\right)=: \mathbf{x}_{Q}^{\mu}-\frac{1}{i \hbar} \alpha^{\nu}\left[\mathbf{x}_{Q}^{\mu}, \mathbf{p}_{Q \nu}\right]
$$

with the usual definition $\sigma_{\nu}^{\mu}:=\sigma^{\mu}{ }_{j \nu}$. We read off the required commutation relations:

$$
\left[\mathbf{x}_{Q}^{\mu}, \mathbf{p}_{Q \nu}\right]=i \hbar \sigma_{\nu}^{\mu}\left(Q, \mathbf{x}_{Q}\right)
$$

The Jacobi identities and $\mathbf{p}_{\nu}=\mathbf{p}_{\nu}^{*}$ then yield

$$
\left[\mathbf{p}_{Q \mu}, \mathbf{p}_{Q \nu}\right]=i \hbar\left\{\mathbf{p}_{Q \alpha}, \sigma_{\rho}^{-1 \alpha} \sigma_{[\nu}^{\epsilon} \sigma_{\mu], \epsilon}^{\rho}\right\}
$$

The Heisenberg algebras $\mathcal{A}_{Q}$ are a special case of Eqs.1,2 so that the path integral in its abstract formulation Eq.11 can be evaluated through Eqs.14-18, as discussed. There exist spectral representations of the $\mathbf{x}_{Q}$ with $\mathbf{p}_{Q \mu} \cdot \psi(x)=-i \hbar\left(1 / 2 \sigma_{\mu, \varepsilon}^{\epsilon}+\sigma_{\mu}^{\epsilon} \partial_{x^{\epsilon}}\right) \psi(x)$. 
Studies into whether $Q$-independence of the action functional can be understood as a gauge principle are in progress.

A detailed comparison of the approach with the conventional treatment is in preparation, and we mention here only a few general points. Path integrals such as Eq.10 are conventionally adapted to the curved space in the position representation, by replacing ordinary derivatives by covariant derivatives and by adapting the measure introducing $\sqrt{|-g|}$, see e.g. [21]. This is in complete analogy to how observable fields would have to be treated. Let us recall however, that for quantum fields this procedure, which singles out position space, spoils the beautiful quantum theoretical representation independence of the action functional. There then no longer exists an algebra $\mathcal{A}$ of positions and momenta, for which one could freely choose a Hilbert space representation. In our approach, we do still have a Heisenberg algebra of positions and momenta, so that the abstract functional analytical structure Eq.11 of the path integral is preserved, and representations of $\mathcal{A}$ need not be position representations, but could as well be a representation e.g. on a Hilbert space of orthogonal polynomials. Note that choosing the position representation the $\mathbf{p}_{i}$ do not act by simple covariant differentiation but by making use of the canonical tensor fields that arise as covariant derivatives $\sigma^{\mu}{ }_{\nu}$ of the world function $\sigma$.

We give the first correction terms explicitly. It is possible to covariantly 'Taylor expand' $\sigma^{\mu}{ }_{\nu}$ within some 'radius' in $U(Q)$, see [23]. To this end $\sigma^{\mu}{ }_{\nu}$ and its higher covariant derivatives are needed in the coincidence limit $Q=x$. As is well known, in the coincidence limit $\sigma_{\nu}^{\mu}(Q, Q)=\delta_{\nu}^{\mu}$, (i.e. also $\left.\sigma_{\mu \nu}(Q, Q)=g_{\mu \nu}(Q)\right)$. The next order term is the torsion tensor $\sigma_{\nu \rho}^{\mu}(Q, Q)=-1 / 2 T^{\mu}{ }_{\nu \rho}(Q)$ which, if we had not taken it to vanish, would contribute a term linear in $\mathbf{x}$ on the RHS of the commutation relation Eq.22. To the second order we have $\sigma_{\nu \rho \tau}^{\mu}(Q, Q)=-1 / 3\left(R_{\rho \nu \tau}^{\mu}(Q)+R^{\mu}{ }_{\tau \nu \rho}(Q)\right)=-2 / 3 J^{\mu}{ }_{\nu \rho \tau}(Q)$, see [23], where the Jacobi curvature tensor is $J^{\mu}{ }_{\nu \alpha \beta}=\frac{1}{2}\left(R_{\alpha \nu \beta}^{\mu}+R_{\beta \nu \alpha}^{\mu}\right)$, see [19]. The covariant Taylor expansion for $\sigma_{\nu}^{\mu}$ then yields

$$
\left[\mathbf{x}_{Q}^{\mu}, \mathbf{p}_{Q \nu}\right]=i \hbar \delta_{\nu}^{\mu}-\frac{i \hbar}{3} J_{\nu \alpha \beta}^{\mu}(Q) \mathbf{x}_{Q}^{\alpha} \mathbf{x}_{Q}^{\beta}+\ldots
$$

and

$$
\left[\mathbf{p}_{Q \mu}, \mathbf{p}_{Q \nu}\right]=\frac{1}{2} i \hbar R_{\alpha \nu \mu}^{\rho}(Q)\left\{\mathbf{x}_{Q}^{\alpha}, \mathbf{p}_{Q \rho}\right\}+\ldots
$$

The higher terms in the covariant expansion of $\sigma^{\mu}{ }_{\nu}$ are expressions in covariant derivatives of $R$, which have been calculated by computer to a few orders, [23].

It is instructive to derive the correction terms also by the pedestrian method, i.e. in coordinates.

Denote by $G_{Q}$ the geodesic coordinate system, or frame, with the event $Q$ at its origin. Further, let the event $Q^{\prime}$ which has the coordinates $\alpha^{\mu}$ in $G_{Q}$ define the origin of a second geodesic frame $G_{Q^{\prime}}$. The axes of $G_{Q^{\prime}}$ be starting off parallel to those of the frame $G_{Q}$, i.e. the corresponding tangent vectors are obtained through parallel transport along the geodesic connecting $Q$ and $Q^{\prime}$.

Under the infinitesimal translation of frames from $Q$ to $Q^{\prime}$ an event's coordinates $x_{Q}^{\mu}$ in $G_{Q}$ transform into it's coordinates $x_{Q^{\prime}}^{\mu}$ in $G_{Q^{\prime}}$ and vice versa, see Eq.21. The coordinates $x_{Q^{\prime}}^{\mu}$ of an event $x$ denote by definition the initial tangent vector of the geodesic $\gamma:[0,1] \rightarrow$ $\mathcal{M}, d / d s \gamma(Q)=x_{Q^{\prime}}^{\mu}, \gamma(0)=Q, \gamma(1)=x$, where in $G_{Q}: \gamma:[0,1] \rightarrow \gamma^{\mu}(s) \in T_{Q}(\mathcal{M})$. The 
end point of this geodesic needs to be calculated in the frame $G_{Q}$ to obtain the coordinates $x_{Q}$ of $x$. The ordinary Taylor theorem yields:

$$
x_{Q}^{\mu}=\left.e^{d / d s} \gamma^{\mu}(s)\right|_{s=0}
$$

In $G_{Q}$ holds $\Gamma(Q)=0$ so that the geodesics through the event $Q^{\prime}$ obey

$$
\frac{d^{2} \gamma^{\mu}}{d s^{2}}(Q)+\Gamma_{\beta_{1} \beta_{2}, \nu}^{\mu}(Q) \alpha^{\nu} \frac{d \gamma^{\beta_{1}}}{d s}(Q) \frac{d \gamma^{\beta_{2}}}{d s}(Q)+\mathcal{O}\left(\alpha^{2}\right)=0
$$

and thus generally to first order in $\alpha$ :

$$
\frac{d^{n+2}}{d s^{n+2}} \gamma^{\mu}(Q)=-\Gamma_{\beta_{1} \beta_{2}, \nu \rho_{1} \ldots \rho_{n}}^{\mu_{1}}(Q) \alpha^{\nu} x_{Q^{\prime}}^{\beta_{1}} x_{Q^{\prime}}^{\beta_{2}} x_{Q^{\prime}}^{\rho_{1}} \ldots x_{Q^{\prime}}^{\rho_{n}}+\mathcal{O}\left(\alpha^{2}\right)
$$

To first nontrivial order the coordinates $x_{Q}^{\mu}$ in the frame $G_{Q}$ of the event with the coordinates $x_{Q^{\prime}}^{\mu}$ in the frame $G_{Q^{\prime}}$ therefore read

$$
x_{Q}^{\mu}=x_{Q^{\prime}}^{\mu}+\alpha^{\mu}-\frac{1}{2} \Gamma_{\alpha \beta, \nu}^{\mu}(Q) \alpha^{\nu} x_{Q^{\prime}}^{\alpha} x_{Q^{\prime}}^{\beta}+\mathcal{O}\left(\alpha^{2}, x_{Q^{\prime}}^{3}\right)
$$

so that

$$
x_{Q^{\prime}}^{\mu}=x_{Q}^{\mu}-\alpha^{\mu}+\frac{1}{2} \Gamma_{\alpha \beta, \nu}^{\mu}(Q) \alpha^{\nu} x_{Q}^{\alpha} x_{Q}^{\beta}+\mathcal{O}\left(\alpha^{2}, x_{Q}^{3}\right)
$$

Hence, with Eq.21:

$$
\sigma_{\nu}^{\mu}(Q, x)=\delta_{\nu}^{\mu}-\frac{1}{2} \Gamma_{\alpha \beta, \nu}^{\mu}(Q) x^{\alpha} x^{\beta}+\mathcal{O}\left(x^{3}\right)
$$

At the origin of geodesic coordinate systems, i.e. here at $Q$, holds [19]: $\Gamma_{\alpha \beta, \nu}^{\mu}(Q)=$ $-\frac{1}{3}\left(R^{\mu}{ }_{\alpha \beta \nu}(Q)+R^{\mu}{ }_{\beta \alpha \nu}(Q)\right)$, so that we recover indeed the above results Eqs.24,25. We also proved that (at the origin of geodesic frames) all $\Gamma_{\nu_{1}\left(\nu_{2}, \nu_{3} \ldots \nu_{r}\right)}(Q)$ are tensors (which has been shown by other methods, inductively in coordinates, in [20]). Note also that from Eq.28 follows $\forall r \geq 2: \Gamma_{\left(\nu_{1} \nu_{2}, \nu_{3} \ldots \nu_{r}\right)}(Q)=0$.

Apart from the suggested physical interpretation, we hope that our approach could provide a useful framework for expressing certain noncommutative geometries as the 'dual' or momentum space to a curved space, and vice versa, thereby possibly making available tools of ordinary geometry for the description of certain noncommutative geometries. Work in this direction is in progress.

Acknowledgement. It is my pleasure to thank the organisers for the very kind hospitality at the Banach Center.

\section{References}

[1] A. Connes, Noncommutative geometry, AP (1994).

[2] P. K. Townsend, Phys. Rev. D, Vol. 15, No 10 2795-2801 (1976).

[3] D. Amati, M. Cialfaloni, G. Veneziano, Phys. Lett. B 216, 41 (1989).

[4] M. Maggiore, Phys. Lett. B 319, 83 (1993).

[5] L. J. Garay, Int. J. Mod. Phys. A10: 145 (1995).

[6] A. Kempf, Lett. Math. Phys. 26: 1-12 (1992).

[7] A. Kempf, J. Math. Phys., Vol. 34, No. 3, 969-987 (1993) . 
[8] A. Kempf, Proc. XXII DGM Conf. Sept. 93 Ixtapa (Mexico), Adv. Appl. Cliff. Alg. (Proc. Suppl. ) (S1) (1994).

[9] A. Kempf, J. Math. Phys. 35 (9): 4483 (1994).

[10] A. Kempf, Preprint DAMTP/94-33, hep-th/9405067, rev. version.

[11] A. Kempf, Czech. J. Phys. (Proc. Suppl. ) 44, 11-12: 1041 (1994).

[12] A. Kempf, G. Mangano, R. B. Mann, Phys. Rev. D52: 1108-1118 (1995).

[13] A. Kempf, H. Hinrichsen, DAMTP/95-50, hep-th/9510144.

[14] A. Kempf, G. Mangano, in preparation.

[15] M. Arik, D. D. Coon, J. Math. Phys. 17: 524 (1975).

[16] W. Pusz, S. Woronowicz, Rep. Math. Phys. 27, 231 (1989).

[17] I. M. Burban, A. U. Klimyk, Lett. Math. Phys. 29, No1: 13 (1993).

[18] R. P. Feynman, Dirac Memorial Lecture, 'The reason for antiparticles' CUP (1987)

[19] C. W. Misner, K. S. Thorne, J. A. Wheeler, Gravitation, p. 285 Freeman \& Co (1973).

[20] Y. Yamashita, Gen. Rel. Grav., 16: 99 (1984).

[21] N. D. Birrell, P. C. W. Davies, Quantum fields in curved space, CUP (1982).

[22] B. S. DeWitt, The Dynamical Theory of Groups and Fields, Gordon \& Breach (1965).

[23] S. A. Fulling, Aspects of Quantum Field Theory in Curved Space-Time, CUP (1989).

[24] S. Doplicher, K. Fredenhagen, J. E. Roberts, Comm. Math. Phys. 172: 187 (1995). 